\title{
Alternative methods for the Plasmodium falciparum artemisinin ring-stage survival assay with increased simplicity and parasite stage-specificity
}

Whitney A. Kite ${ }^{1}$, Viviana A. Melendez-Muniz ${ }^{1,2}$, Roberto R. Moraes Barros ${ }^{1}$, Thomas E. Wellems ${ }^{1}$ and Juliana M. Sá ${ }^{*}$

\begin{abstract}
Background: Artemisinin-based combination therapy is recommended to treat Plasmodium falciparum worldwide, but observations of longer artemisinin (ART) parasite clearance times (PCTs) in Southeast Asia are widely interpreted as a sign of potential ART resistance. In search of an in vitro correlate of in vivo PCT after ART treatment, a ring-stage survival assay (RSA) of $0-3 \mathrm{~h}$ parasites was developed and linked to polymorphisms in the Kelch propeller protein (K13). However, RSA remains a laborious process, involving heparin, Percoll gradient, and sorbitol treatments to obtain rings in the $0-3 \mathrm{~h}$ window. Here two alternative RSA protocols are presented and compared to the standard Percollbased method, one highly stage-specific and one streamlined for laboratory application.

Methods: For all protocols, P. falciparum cultures were synchronized with $5 \%$ sorbitol treatment twice over two intraerythrocytic cycles. For a filtration-based RSA, late-stage schizonts were passed through a $1.2 \mu \mathrm{m}$ filter to isolate merozoites, which were incubated with uninfected erythrocytes for $45 \mathrm{~min}$. The erythrocytes were then washed to remove lysis products and further incubated until $3 \mathrm{~h}$ post-filtration. Parasites were pulsed with either $0.1 \%$ dimethyl sulfoxide (DMSO) or $700 \mathrm{nM}$ dihydroartemisinin in $0.1 \%$ DMSO for $6 \mathrm{~h}$, washed twice in drug-free media, and incubated for 66-90 h, when survival was assessed by microscopy. For a sorbitol-only RSA, synchronized young (0-3 h) rings were treated with $5 \%$ sorbitol once more prior to the assay and adjusted to $1 \%$ parasitaemia. The drug pulse, incubation, and survival assessment were as described above.
\end{abstract}

Results: Ring-stage survival of P. falciparum parasites containing either the K13 C580 or C580Y polymorphism (associated with low and high RSA survival, respectively) were assessed by the described filtration and sorbitol-only methods and produced comparable results to the reported Percoll gradient RSA. Advantages of both new methods include: fewer reagents, decreased time investment, and fewer procedural steps, with enhanced stage-specificity conferred by the filtration method.

Conclusions: Assessing P. falciparum ART sensitivity in vitro via RSA can be streamlined and accurately evaluated in the laboratory by filtration or sorbitol synchronization methods, thus increasing the accessibility of the assay to research groups.

Keywords: Plasmodium falciparum, Artemisinin, In vitro anti-malarial assays

\footnotetext{
*Correspondence: jsa@niaid.nih.gov

${ }^{1}$ Laboratory of Malaria and Vector Research, National Institute of Allergy and Infectious Diseases, National Institutes of Health, 12735 Twinbrook Parkway, Room 3E - 10, Rockville, MD 20852, USA

Full list of author information is available at the end of the article
} 


\section{Background}

In accordance with the World Health Organization (WHO) guidelines, artemisinin-based combination therapy (ACT) is now the front-line treatment for Plasmodium falciparum [1]. Increased awareness of prolonged parasite clearance times (PCTs) following artemisinin (ART) treatment in the Greater Mekong subregion has raised concern of potential ART resistance [2-4]. Biological studies of the PCTs and their in vitro surrogates will improve understanding of the mechanisms involved and their impact on parasite fitness $[5,6]$.

Unlike typical anti-malarial resistance, delayed parasite clearance is not associated with a substantial change in ART $50 \%$ inhibitory concentrations $\left(\mathrm{IC}_{50}\right)[3,7,8]$, evaluated by exposing parasites in vitro to serial dilutions of drug $[9,10]$. In vitro studies have shown that response to anti-malarials, such as ART, is largely dependent upon intra-erythrocytic parasite stage, as trophozoites and schizonts prove highly susceptible to ART regardless of in vivo phenotype $[11,12]$. However, ring-stage parasites aged $0-3 \mathrm{~h}$ show differential ability to survive pulses of ART in vitro among genetically distinct parasites $[8,13$, 14]. Accordingly, a ring-stage survival assay (RSA) was developed to distinguish parasite survival of an ART drug pulse in vitro. Use of the RSA led to the association of in vitro survival, prolonged PCT, and parasite polymorphisms in Kelch propeller protein (K13) [15, 16]. While ACT is still efficacious, resistance to partner drugs and ART monotherapy place increasing pressure on ART [17], thereby necessitating efforts to investigate the developing relationship of RSAs, K13 polymorphisms, PCTs, and ACT anti-malarial efficacy as thoroughly as possible [18].

RSAs generally rely on a method using heparin, a Percoll gradient, and sorbitol to enrich for $0-3 \mathrm{~h}$ ring-stages. Rings are then pulsed with $700 \mathrm{nM}$ dihydroartemisinin (DHA, active metabolite of ART) for $6 \mathrm{~h}$, washed, and grown for $66 \mathrm{~h}$, at which time survival is assessed by microscopy [8]. While effective, this protocol includes multiple steps over many hours, involving several chemical preparations and significant volumes of parasites. Here two alternative RSA preparations are presented: one focused on higher stage-specificity and another on increased time and resource efficiency. The first uses the precision of schizont filtration to isolate merozoites [19], which, after incubating with erythrocytes (red blood cells, RBCs), result in a highly synchronous $3 \mathrm{~h}$ ring-stage culture. The second uses sorbitol synchronization only and significantly lessens time investment, thus reducing the effort of the RSA procedure and allowing several assays to be done in parallel with ease. Accurately and efficiently assessing parasites at a specific age supports investigations to understand ART's mechanism of action and the ability of Plasmodium to develop resistance.

\section{Methods \\ Parasites}

To evaluate RSA methods $P$. falciparum lines carrying the K13 propeller polymorphism C580Y, which is associated with prolonged PCT and approximately $10 \%$ RSA survival [15, 16], and K13 propeller C580 lines, known to have less than $1 \%$ viable parasites after RSA [8], were chosen. Parasite line 803 contains the K13 C580Y polymorphism and was isolated from a Cambodian patient presenting an extended PCT while treated with three daily oral doses of $4 \mathrm{mg} / \mathrm{kg}$ artesunate (AS) [7]. Clone GB4, which does not contain any polymorphisms in the Kelch propeller domain associated with increased PCTs or RSA survival (K13 C580), was isolated from Ghana prior to widespread use of ART in Africa [20]. Progeny from a genetic cross between 803 and GB4 containing either K13 C580 (clones 36F11, 46G9, 24G11, 39E5, and 34F5) or K13 C580Y (clones 61E8 and 76H10) were included in the analysis, along with the parental lines.

\section{Culture conditions}

Parasite cultures were maintained at $5 \%$ haematocrit between $0.5-3.0 \%$ parasitaemia, in complete media (cRPMI): RPMI-1640 (KD Medical, MD, USA) containing $25 \mathrm{mM}$ HEPES and $50 \mu \mathrm{g} / \mathrm{mL}$ hypoxanthine, supplemented with $0.21 \%$ sodium bicarbonate, $20 \mathrm{mg} / \mathrm{L}$ gentamicin, and $1 \%$ Albumax II (Life Technologies, CA, USA). Cultures were incubated at $37{ }^{\circ} \mathrm{C}$ under a $90 \% \mathrm{~N}_{2}, 5 \% \mathrm{CO}_{2}$, and $5 \% \mathrm{O}_{2}$ gas mixture. Erythrocytes were purchased from the Interstate Blood Bank (Memphis, TN, USA), passed through a Sepacell R-500 filter (Baxter, Deerfield, IL, USA) to remove leukocytes and platelets, washed, re-suspended in RPMI 1640 medium, and stored at $4{ }^{\circ} \mathrm{C}$ until use.

\section{Percoll gradient ring-stage survival assay}

To perform the 0-3 h RSA protocol developed by Witkowski, Amaratunga, and colleagues [8] (henceforth designated as the Percoll gradient RSA), $300 \mu \mathrm{L}$ of packed parasitized RBCs containing $2-3 \%$ mature schizonts $(>10$ well-segmented nuclei) were collected from a P. falciparum culture previously synchronized twice with $5 \%$ sorbitol (Sigma-Aldrich, MO, USA) dissolved in distilled tissue culture-grade water (Gibco, MD, USA), $10 \mathrm{~min}, 37{ }^{\circ} \mathrm{C}$, over two parasite intra-erythrocytic cycles. The parasites were then incubated in RPMI-1640 containing $15 \mathrm{U} / \mathrm{mL}$ heparin (Sigma-Aldrich), but no other supplements, for $15 \mathrm{~min}$ at $37{ }^{\circ} \mathrm{C}$. After incubation, parasites were placed onto a 35/65 \% Percoll (GE Healthcare Life Sciences, Pittsburgh, PA, USA) gradient, centrifuged at $3000 \mathrm{rpm}$ 
for $10 \mathrm{~min}(966 \times g$, Eppendorf Centrifuge 5810, rotor A-481 , no brake). The inter-phase band of late-stage schizonts was collected and incubated in media with fresh RBCs for $3 \mathrm{~h}$, at $37{ }^{\circ} \mathrm{C}$ under $90 \% \mathrm{~N}_{2}, 5 \% \mathrm{CO}_{2}$, and $5 \% \mathrm{O}_{2}$ gas mixture (see Additional file 1). This gradient is slightly modified from the original Percoll gradient RSA, as per recommendation of Chanaki Amaratunga to improve separation (personal communication). After incubation, some schizonts had yet to invade; thus, parasites were treated with $5 \%$ sorbitol again to remove remaining schizonts and isolate $0-3 \mathrm{~h}$ ring-stage parasites. The parasite mixture was adjusted to $1 \%$ parasitaemia in $20 \mu \mathrm{l}$ with fresh packed RBCs, and suspended in $1 \mathrm{~mL}$ of cRPMI in a 24-well plate containing either $0.1 \%$ dimethyl sulfoxide (DMSO, Sigma-Aldrich, St. Louis, MO, USA) or $700 \mathrm{nM}$ DHA (Sigma-Aldrich, MO, USA) dissolved in $0.1 \%$ DMSO for $6 \mathrm{~h}$. After drug pulse, cells were washed twice with $10 \mathrm{~mL}$ cRPMI with $3 \mathrm{~min}$ centrifugation at $2500 \mathrm{rpm}$ $(671 \times g$, 9 acceleration, 5 de-acceleration, Eppendorf Centrifuge 5810, rotor A-4-81). Parasites were further incubated with drug-free cRPMI for $66 \mathrm{~h}$, when thin blood smears were prepared, methanol fixed, and stained with 20 \% Giemsa (Sigma-Aldrich, MO, USA) for $15 \mathrm{~min}$ for independent survival assessment by at least two experienced microscopists. Evaluation consisted of counting the number of parasitized cells in an estimated 10,000 RBCs and comparing survival to DMSO drug-free incubation.

\section{Filtration ring-stage survival assay}

Parasite cultures were twice synchronized with $5 \%$ sorbitol over two parasite cycles and grown to $40 \mathrm{~mL}$ of $5 \%$ haematocrit cultures with at least $2 \%$ parasitaemia. When the majority of parasites were late-stage schizonts, judged as overtly segmented and within minutes of bursting (Fig. 1), cultures were collected, centrifuged at $2500 \mathrm{rpm}$ for $3 \mathrm{~min}(671 \times g, 9$ acceleration, $5 \mathrm{de}-$ acceleration, Eppendorf Centrifuge 5810, rotor A-4-81), combined, and suspended in $10 \mathrm{~mL}$ cRPMI. After transferring to a $12 \mathrm{~mL}$ syringe, parasites were pushed through a $1.2 \mu \mathrm{m}$ filter (Pall Corporation, Port Washington, NY, USA) into 200-250 $\mu \mathrm{L}$ fresh RBCs (packed cells) in $10 \mathrm{~mL}$ fresh cRPMI [19]. After filtration, the mixture was incubated for $45 \mathrm{~min}$ on a plate shaker (speed 3, Boekel Scientific Orbritron V, model 281111), with a $90 \% \mathrm{~N}_{2}$, $5 \% \mathrm{CO}_{2}$, and $5 \% \mathrm{O}_{2}$ gas mixture at $37^{\circ} \mathrm{C}$. Cells were then washed with $10 \mathrm{~mL}$ cRPMI with 3 min centrifugation at $2500 \mathrm{rpm}(671 \times g, 9$ acceleration, 5 de-acceleration, Eppendorf Centrifuge 5810, rotor A-4-81) to remove remnants of lysed parasites and RBCs, then further incubated until $3 \mathrm{~h}$ post-filtration. Resulting $3 \mathrm{~h}$ ring cultures of $0.07-0.15 \%$ initial parasitaemia were adjusted to $2 \%$ haematocrit in $1 \mathrm{~mL}$ cRPMI in a 24-well plate containing either $0.1 \%$ DMSO or $700 \mathrm{nM}$ DHA in $0.1 \%$ DMSO and further incubated at $37^{\circ} \mathrm{C}$ with gas conditions above (Fig. 2a). After $6 \mathrm{~h}$ incubation, cells were washed twice in $10 \mathrm{~mL}$ drug-free cRPMI, and then placed in new wells with $1 \mathrm{~mL}$ fresh cRPMI for $66 \mathrm{~h}$ cultivation. After incubation, thin blood smears of each well were methanol fixed and stained with $20 \%$ Giemsa for 15 min, coded for "blind" parasitaemia assessment, and counted independently by two microscopists, with percent survival adjusted to the DMSO parasitaemia (by counting the number of parasitized cells in estimated 10,000 RBCs). Given the precise age necessary for survival of merozoite filtration, the majority of parasites perish in the filtering process. When too few parasites survive filtration,
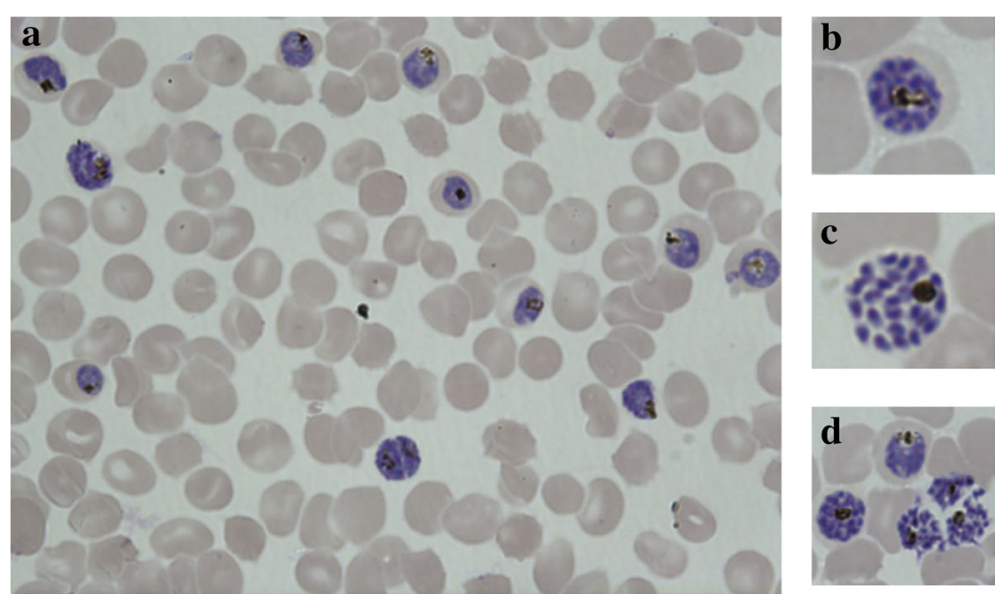

Fig. 1 Target morphology of segmented schizonts for filtration. a Cultures (1-2 mL packed RBCs) need to be at least 2-3\% mature schizonts for filtration method to work efficiently. b-d Schizont nuclei are distinct and separated prior to filtration, allowing parasites to burst upon passing through the filter and enter the assay as viable merozoites 


\section{$\mathbf{a}$}

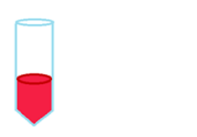

Sorbitol synchronized segmented schizonts

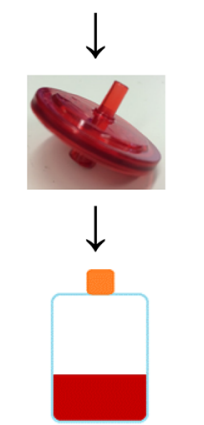

Incubate $45 \mathrm{~min}$

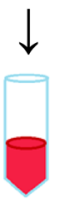

Wash off lysed cells

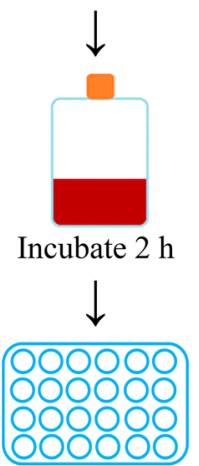

Incubate for $6 \mathrm{~h}$ with

DMSO/DHA; wash cells;

incubate for $66-90 \mathrm{~h}$

Fig. 2 Preparations for drug pulse in filtration and sorbitol-only assays. a Steps for filtration RSA are depicted. Late-stage, segmented schizonts are collected, pushed through a $1.2 \mu \mathrm{m}$ filter, incubated, washed, incubated again, and finally incubated with either $700 \mathrm{nM}$ DHA in $0.1 \%$ DMSO or 0.1 \% DMSO only. b Sorbitol-only RSA involves a single step. Early post-invasion rings (approximately $3 \mathrm{~h}$ old) are collected, synchronized with sorbitol to remove remaining schizonts, and plated at $1 \%$ parasitaemia with either $700 \mathrm{nM} \mathrm{DHA}$ in $0.1 \%$ DMSO or $0.1 \%$ DMSO

only low parasitaemias are achieved by $66 \mathrm{~h}$ incubation, even in DMSO control wells. In cases of particularly low DMSO parasitaemia $(<0.40 \%)$, the cultures were incubated with fresh cRPMI for an additional $24 \mathrm{~h}$ and final smears were made at the $96 \mathrm{~h}$ time point.

\section{Sorbitol-only ring-stage survival assay}

Parasite cultures were synchronized with $5 \%$ sorbitol twice at exactly $46 \mathrm{~h}$ intervals to tighten synchronicity. When parasites were between very late schizonts and very early rings, cultures were synchronized with $5 \%$ sorbitol once more to remove remaining mature forms. Immediately after sorbitol treatment, parasite cultures were adjusted to $1 \%$ parasitaemia in $20 \mu \mathrm{L}$ RBCs in $1 \mathrm{~mL}$ of cRPMI in a 24-well plate, with either $0.1 \%$ DMSO or $700 \mathrm{nM}$ DHA dissolved in $0.1 \%$ DMSO (Fig. 2b). After $6 \mathrm{~h}$ incubation, cells were washed and incubated with drug-free cRPMI for $66 \mathrm{~h}$, after which thin blood smears were prepared, methanol fixed, stained with $20 \%$ Giemsa for $15 \mathrm{~min}$ and read by at least two microscopists. Percent survival was calculated by counting the number of parasitized cells in estimated 10,000 RBCs and comparing survival to that of the drug-free DMSO incubation.

\section{Results and discussion}

As shown in Fig. 3, all three methods produce comparable results, distinguishing survival of parasites with K13 C580 and C580Y. With the Percoll gradient and sorbitolonly methods, $<1-2 \%$ of K13 C580 parasites are viable after the DHA pulse, compared to $6-15 \%$ of K13 C580Y parasites. With the filtration method, the ranges of survival increase, with $<1-3 \%$ of K13 C580 parasites and as much as $20 \%$ of K13 C580Y parasites persisting after the DHA pulse. Across all three RSA methods, the distinction between high and low survival remains clear.

With all three methods producing consistent outcomes, the methodologies can be directly compared for their respective benefits. Table 1 delineates the distinguishing features of each of the three methods. The two alternative methods presented here eliminate the need for heparin and Percoll. While the required number of parasitized cells is increased fivefold in the filtration protocol, it is reduced tenfold with the sorbitol-only RSA. The filtration RSA may include an additional $24 \mathrm{~h}$ incubation in the case of a low initial parasitaemia; however, in response to a significant burden of conducting RSAs, the time required to set up each assay is decreased in both alternative methods.

The variation observed between experiments involves several possible confounding factors. In the Percoll and sorbitol-only protocols, variation can be attributed to older rings and even some mature stages surviving $5 \%$ sorbitol synchronization; older rings of sensitive lines have been shown to have decreased sensitivity, and mature stages of both K13 C580 and C580Y lines are highly sensitive to DHA in vitro, thus potentially skewing results $[4,8]$. Despite the heightened stage-specificity of the filtration RSA, variation between experiments 


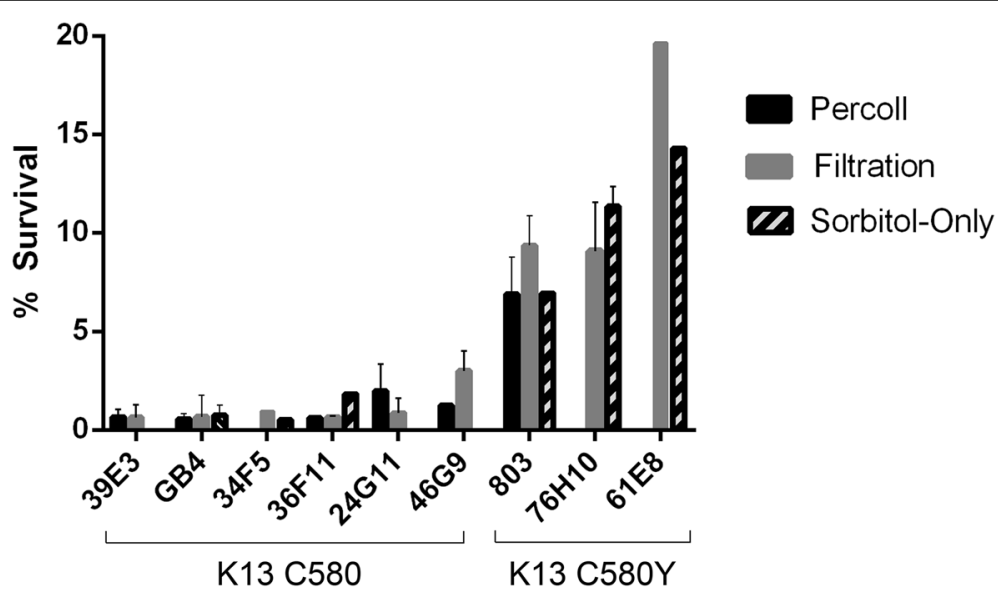

Fig. 3 Comparison of results obtained with three RSA methods. The Percoll method is shown in black, the filtration method in gray, and the sorbitol-only method in pattern. Across the three methods, results are consistent for each line and the distinction between sensitive and surviving is clear. GB4 and progeny 39E3, 34F5, 36F11, and 24G11 all contain K13 C580, while 803 and progeny $76 \mathrm{H} 10$ and $61 \mathrm{E} 8$ contain K13 C580Y. Survival of all K13 C580 lines is significantly less than those containing the K13 C580Y polymorphism. Values are presented in detail in Additional file 2

Table 1 Comparison of RSA protocols

\begin{tabular}{|c|c|c|c|}
\hline Variables & Percoll gradient RSA [8] & Sorbitol-only RSA & Filtration RSA \\
\hline Chemicals required during $\mathrm{RSA}^{\mathrm{a}}$ & Heparin, percoll, sorbitol & Sorbitol & None \\
\hline Volume of parasite culture needed & $\begin{array}{l}\geq 300 \mu \mathrm{L} \text { packed } \mathrm{RBCs} \\
\text { at } 2 \% \text { parasitemia }\end{array}$ & $\begin{array}{l}40 \mu \mathrm{L} \text { packed } \mathrm{RBCs} \text { at } \\
1 \% \text { parasitemia }\end{array}$ & $\begin{array}{l}\geq 1.5 \mathrm{~mL} \text { packed RBCs at } 2-3 \% \\
\text { parasitemia }\end{array}$ \\
\hline Number of steps until completion & 17 & 10 & 12 \\
\hline Preparation time, before drug pulse & $4.5 \mathrm{~h}$ & $30 \mathrm{~min}$ & $3 h$ \\
\hline $\begin{array}{l}\text { Total time of parasite incubation } \\
\text { from drug pulse }\end{array}$ & $66 \mathrm{~h}$ & $66 \mathrm{~h}$ & 66 or $90 \mathrm{~h}$, if DMSO $<0.40 \%$ at $66 \mathrm{~h}$ \\
\hline $\begin{array}{l}\text { Stage distribution at time of } \\
\text { drug pulse }\end{array}$ & $\begin{array}{l}80 \% \text { rings, } 20 \% \text { trophozoites } \\
\text { and schizonts }\end{array}$ & $\begin{array}{l}\text { Mixture of young } \\
\text { and older rings }\end{array}$ & $3 \mathrm{~h}$ rings only \\
\hline $\begin{array}{l}\text { Presence of pyknotic parasites } \\
\text { in read out }\end{array}$ & Yes & Yes & No \\
\hline
\end{tabular}

a All methods require sorbitol synchronization two parasite cycles before performing RSA

remains similar to the other methods. This variation could occur because of invasion variability in the 66-90 h post-drug pulse incubation. Measuring survival $24 \mathrm{~h}$ after drug pulse would eliminate this variable; however, as the initial parasitaemia of the filtration method can be as low as $0.07 \%$, measuring survival prior to one or even two rounds of invasion can be difficult.

The large number of pyknotic forms present in the $72 \mathrm{~h}$ smears after using the Percoll or sorbitol-only methods may further confound results. Judgment of the viability of the forms by microscopy introduces an additional level of uncertainty and room for error. However, the use of flow cytometry for determination of percent survival may help address this issue [4]. For laboratories without access to flow cytometry, the filtration method eases microscopy assessment as no pyknotic forms are present in the final smears. A possible explanation based on the homogeneity of age distribution suggests highly sensitive and $3 \mathrm{~h}$ tightly synchronized rings may simply lyse after drug exposure, whereas slightly older rings present in Percoll or sorbitol-based methods may become pyknotic. These features offer confidence in the results produced by the filtration method, as there is less room for error both in the stage of the exposed parasites and in microscopy assessment.

Others have addressed the need for optimization through highly time-sensitive sorbitol synchronizations [21]. However, while the involved synchronization improves start times for the Percoll assay, it still requires large time investments with over a week of long, irregular hours. In contrast, the proposed alternatives decrease time and resource investment. With the sorbitol-only method, time investment per assay can be decreased to as little as $30 \mathrm{~min}$ from collecting parasites to starting the drug pulse. 
The simpler setup of the assay allows multiple lines to be tested simultaneously with ease, thereby increasing the efficiency of the protocol even further. The $46 \mathrm{~h}$ spacing of sorbitol synchronizations is based on the specific in vitro cell cycle of the lines presented here and may not suit the cell cycles of other isolates. Individual laboratories should address the timing of synchronization to align with the cell cycles of their isolates and prevent selection of older rings from isolates with shorter cycles.

Apart from improving RSA stage-specificity, the potential precision of the filtration method opens the door to wider applications of the assay. ART's mechanism of action may be related to cell stress responses and delayed cell cycles, investigated through time-specific drug pulses and carefully tracking parasite growth after ART exposure [14, 22]. For these assays, accurately staging parasites and maintaining a high level of synchronicity is essential. While estimates of delayed growth are possible through sorbitol synchronized parasites, the stage-specificity of the filtration RSA provides an accurate platform upon which to examine growth-related phenomenon.

\section{Conclusions}

The alternative RSA protocols described here provide simplicity and specificity without compromising the results. The filtration method provides greater stagespecificity, thereby ensuring the age of the parasites is consistent across experiments. The sorbitol-only method provides an alternative to the original method with fewer chemicals, less time, and one-tenth of the parasite culture volume. These methods have not been tested outside the laboratory and thus will need to be validated against the Percoll gradient method in field settings. However, for testing laboratory adapted lines in vitro, the alternative methods provide an opportunity to conduct RSAs with ease and efficiency, thus allowing laboratories with limited time and resources to study ART response in vitro where previously impractical.

\section{Additional files}

Additional file 1. P. falciparum late-stage schizont enrichment from blood cultures using 35/65 \% Percoll gradient.

Additional file 2. Comparison of RSA results by parasite clone.

\footnotetext{
Abbreviations

WHO: World Health Organization; ART: artemisinin and its derivatives; ACT: artemisinin-based combination therapy; PCT: parasite clearance time; IC- ${ }_{50}$ : $50 \%$ inhibitory concentration; h: hour(s); RSA: ring-stage survival assay; K13: Kelch propeller protein; DHA: dihydroartemisinin; CRPMI: complete RPMI 1640 media; RBCs: erythrocytes; min: minutes; DMSO: dimethyl sulfoxide; C: cysteine; $Y$ : tyrosine.
}

\section{Authors' contributions}

WAK and JMS designed and performed experiments, and wrote the manuscript. VAMM and RRMB performed experiments and reviewed the manuscript. TEW designed experiments and contributed to writing the manuscript. All authors read and approved the final manuscript.

\section{Author details}

${ }^{1}$ Laboratory of Malaria and Vector Research, National Institute of Allergy and Infectious Diseases, National Institutes of Health, 12735 Twinbrook Parkway, Room 3E - 10, Rockville, MD 20852, USA. ${ }^{2}$ Universidad Central del Caribe School of Medicine, Bayamon, PR 00960, USA

\section{Acknowledgements}

We thank Paul Han, Michael A. Krause, and Anna Liu for providing support with culturing and setting up experiments, Chanaki Amaratunga for hands on training in the Percoll gradient RSA, and Jennifer S. Armistead, Tyler Gibson, and J. Patrick Mershon for providing feedback on the manuscript. The Intramural Research Program of the National Institute of Allergy and Infectious Disease, National Institutes of Health supported this research.

\section{Competing interests}

The authors declare that they have no competing interests.

Received: 10 November 2015 Accepted: 4 February 2016 Published online: 17 February 2016

\section{References}

1. WHO. Guidelines for the treatment of malaria. 3rd ed. Geneva: World Health Organization; 2015.

2. Noedl H, Se Y, Schaecher K, Smith BL, Socheat D, Fukuda MM, et al. Evidence of artemisinin-resistant malaria in western Cambodia. N Engl J Med. 2008;359:2619-20.

3. Dondorp AM, Nosten F, Yi P, Das D, Phyo AP, Tarning J, et al. Artemisinin resistance in Plasmodium falciparum malaria. N Engl J Med. 2009;361:455-67.

4. Amaratunga C, Witkowski B, Khim N, Menard D, Fairhurst RM. Artemisinin resistance in Plasmodium falciparum. Lancet Infect Dis. 2014;14:449-50.

5. Takala-Harrison S, Clark TG, Jacob CG, Cummings MP, Miotto O, Dondorp AM, et al. Genetic loci associated with delayed clearance of Plasmodium falciparum following artemisinin treatment in Southeast Asia. Proc Natl Acad Sci USA. 2013;110:240-5.

6. Hastings IM, Kay K, Hodel EM. How robust are malaria parasite clearance rates as indicators of drug effectiveness and resistance? Antimicrob Agents Chemother. 2015;59:6428-36.

7. Amaratunga C, Sreng S, Suon S, Phelps ES, Stepniewska K, Lim P, et al. Artemisinin-resistant Plasmodium falciparum in Pursat province, western Cambodia: a parasite clearance rate study. Lancet Infect Dis. 2012;12:851-8.

8. Witkowski B, Amaratunga C, Khim N, Sreng S, Chim P, Kim S, et al. Novel phenotypic assays for the detection of artemisinin-resistant Plasmodium falciparum malaria in Cambodia: in vitro and ex vivo drug-response studies. Lancet Infect Dis. 2013;13:1043-9.

9. Desjardins RE, Canfield CJ, Haynes JD, Chulay JD. Quantitative assessment of antimalarial activity in vitro by a semiautomated microdilution technique. Antimicrob Agents Chemother. 1979;16:710-8.

10. Smilkstein M, Sriwilaijaroen N, Kelly JX, Wilairat P, Riscoe M. Simple and inexpensive fluorescence-based technique for high-throughput antimalarial drug screening. Antimicrob Agents Chemother. 2004;48:1803-6.

11. ter Kuile F, White NJ, Holloway P, Pasvol G, Krishna S. Plasmodium falciparum: in vitro studies of the pharmacodynamic properties of drugs used for the treatment of severe malaria. Exp Parasitol. 1993;76:85-95.

12. Witkowski B, Lelievre J, Barragan MJ, Laurent V, Su XZ, Berry A, et al. Increased tolerance to artemisinin in Plasmodium falciparum is mediated by a quiescence mechanism. Antimicrob Agents Chemother. 2010;54:1872-7. 
13. Klonis N, Xie SC, McCaw JM, Crespo-Ortiz MP, Zaloumis SG, Simpson JA, et al. Altered temporal response of malaria parasites determines differential sensitivity to artemisinin. Proc Natl Acad Sci USA. 2013;1 10:5157-62.

14. Dogovski C, Xie SC, Burgio G, Bridgford J, Mok S, McCaw JM, et al. Targeting the cell stress response of Plasmodium falciparum to overcome artemisinin resistance. PLoS Biol. 2015;13:e1002132.

15. Ariey F, Witkowski B, Amaratunga C, Beghain J, Langlois AC, Khim N, et al. A molecular marker of artemisinin-resistant Plasmodium falciparum malaria. Nature. 2014;505:50-5.

16. Straimer J, Gnadig NF, Witkowski B, Amaratunga C, Duru V, Ramadani AP, et al. Drug resistance. K13-propeller mutations confer artemisinin resistance in Plasmodium falciparum clinical isolates. Science. 2015;347:428-31.

17. Li GQ, Arnold K, Guo XB, Jian HX, Fu LC. Randomised comparative study of mefloquine, qinghaosu, and pyrimethamine-sulfadoxine in patients with falciparum malaria. Lancet. 1984;2:1360-1.

18. Ashley EA, Dhorda M, Fairhurst RM, Amaratunga C, Lim P, Suon S, et al. Spread of artemisinin resistance in Plasmodium falciparum malaria. N Engl J Med. 2014;371:411-23.
19. Boyle MJ, Wilson DW, Richards JS, Riglar DT, Tetteh KK, Conway DJ, et al. Isolation of viable Plasmodium falciparum merozoites to define erythrocyte invasion events and advance vaccine and drug development. Proc Natl Acad Sci USA. 2010;107:14378-83.

20. Sullivan JS, Sullivan JJ, Williams A, Grady KK, Bounngaseng A, Huber CS, et al. Adaptation of a strain of Plasmodium falciparum from Ghana to Aotus lemurinus griseimembra, A. nancymai, and A. vociferans monkeys. Am J Trop Med Hyg. 2003;69:593-600.

21. Xie SC, Dogovski C, Kenny S, Tilley L, Klonis N. Optimal assay design for determining the in vitro sensitivity of ring stage Plasmodium falciparum to artemisinins. Int J Parasitol. 2014;44:893-9.

22. Hott A, Casandra D, Sparks KN, Morton LC, Castanares GG, Rutter A, et al. Artemisinin-resistant Plasmodium falciparum parasites exhibit altered patterns of development in infected erythrocytes. Antimicrob Agents Chemother. 2015;59:3156-67.

\section{Submit your next manuscript to BioMed Central and we will help you at every step:}

- We accept pre-submission inquiries

- Our selector tool helps you to find the most relevant journal

- We provide round the clock customer support

- Convenient online submission

- Thorough peer review

- Inclusion in PubMed and all major indexing services

- Maximum visibility for your research

Submit your manuscript at www.biomedcentral.com/submit

() Biomed Central 